\title{
SkpA Restrains Synaptic Terminal Growth during Development and Promotes Axonal Degeneration following Injury
}

\author{
E. J. Brace, ${ }^{1}$ Chunlai Wu, ${ }^{2}$ Vera Valakh, ${ }^{1}$ and ${ }^{\oplus}$ Aaron DiAntonio ${ }^{1}$ \\ ${ }^{1}$ Department of Developmental Biology, Washington University School of Medicine, St Louis, Missouri 63110, and 2Neuroscience Center of Excellence, \\ Louisiana State University Health Sciences Center, New Orleans, Louisiana 70112
}

The Wallenda (Wnd)/dual leucine zipper kinase (DLK)-Jnk pathway is an evolutionarily conserved MAPK signaling pathway that functions during neuronal development and following axonal injury. Improper pathway activation causes defects in axonal guidance and synaptic growth, whereas loss-of-function mutations in pathway components impairs axonal regeneration and degeneration after injury. Regulation of this pathway is in part through the E3 ubiquitin ligase Highwire (Hiw), which targets Wnd/DLK for degradation to limit MAPK signaling. To explore mechanisms controlling Wnd/DLK signaling, we performed a large-scale genetic screen in Drosophila to identify negative regulators of the pathway. Here we describe the identification and characterization of SkpA, a core component of SCF E3 ubiquitin ligases. Mutants in SkpA display synaptic overgrowth and an increase in Jnk signaling, similar to hiw mutants. The combination of hypomorphic alleles of $S k p A$ and hiw leads to enhanced synaptic growth. Mutants in the Wnd-Jnk pathway suppress the overgrowth of $S k p A$ mutants demonstrating that the synaptic overgrowth is due to increased Jnk signaling. These findings support the model that SkpA and the E3 ligase Hiw function as part of an SCF-like complex that attenuates Wnd/DLK signaling. In addition, SkpA, like Hiw, is required for synaptic and axonal responses to injury. Synapses in $S k p A$ mutants are more stable following genetic or traumatic axonal injury, and axon loss is delayed in SkpA mutants after nerve crush. As in highwire mutants, this axonal protection requires Nmnat. Hence, SkpA is a novel negative regulator of the Wnd-Jnk pathway that functions with Hiw to regulate both synaptic development and axonal maintenance.

Key words: axonal injury response; Jnk signaling; MAP kinase; Nmnat; PHR ligase

\section{Introduction}

The growth, activity, and maintenance of synapses are vital for the proper formation and function of neuronal circuits. The Highwire-Wallenda-Jnk pathway (Hiw-Wnd-Jnk) plays a key role in each of these processes. This pathway was first identified due to its role in regulating synaptic growth and development, and more recently has been demonstrated to be a key determinant of the axonal response to injury.

Drosophila Wallenda and its mammalian homolog dual leucine zipper kinase (DLK) are members of the mixed-lineage kinase family and function as MAP Kinase Kinase Kinases (MAP-

Received Nov. 6, 2013; revised April 22, 2014; accepted May 13, 2014.

Author contributions: E.J.B. and A.D. designed research; E.J.B., C.W., A.D., and V.V. performed research; E.J.B. and A.D. contributed unpublished reagents/analytic tools; E.J.B. and A.D. analyzed data; E.J.B. and A.D. wrote the paper.

This work was supported by Grants from NIH (DA020812 and NS065053) and the Morton Cure Paralysis Fund (A.D.), as well as a postdoctoral research award from the American Heart Association (E.J.B.). We thank Xiaolu Sun for technical assistance on the project; Yogi Wairkar, Richard Daniels, Jung Eun Shin and the rest of the DiAntonio laboratory for insightful discussions and comments on this project and paper; Terence Murphy, the Bloomington Stock Center at the University of Indiana, and the Vienna Drosophila RNAi Center for numerous stocks; the Developmental Studies Hybridoma Bank at the University of lowa for antibodies; the Drosophila Genomics Resource Center (DGRC) for DNA clones; and Paul Cliften at the Genome Technology Access Center at Washington University for help with whole genome sequencing and bioinformatics.

The authors declare no competing financial interests.

Correspondence should be addressed to Dr Aaron DiAntonio, Washington University School of Medicine, Campus Box 8103, 660 South Euclid, WUMC, St Louis, M0 63110. E-mail: diantonio@wustl.edu.

DOI:10.1523/JNEUROSCI.4715-13.2014

Copyright $\odot 2014$ the authors $\quad 0270-6474 / 14 / 348398-13 \$ 15.00 / 0$
KKKs) to activate Jnk signaling. Hiw is the Drosophila homolog of the PHR family of E3 ubiquitin ligases (human Pam, mouse Phr1, zebrafish Esrom, and Caenorhabditis elegans RPM-1) that target substrates for ubiquitination and degradation (Schaefer et al., 2000; Wan et al., 2000; Zhen et al., 2000; Po et al., 2010). In the absence of Hiw, Wnd levels are elevated resulting in overactivation of the Jnk pathway and an increase in synaptic growth (Nakata et al., 2005; Collins et al., 2006).

Hiw and DLK/Wnd function not only in synaptic development, but also in response to axonal injury. DLK/Wnd is required for axonal regeneration in Drosophila, C. elegans, and mice (Hammarlund et al., 2009; Yan et al., 2009; Xiong et al., 2010; Shin et al., 2012; Watkins et al., 2013). In addition, mutants in $d l k$ or inhibitors of Jnk result in a delay in axonal degeneration in both fly and mouse models of Wallerian degeneration (Miller et al., 2009; Yoshimura et al., 2011), as well as models of glaucoma (Watkins et al., 2013; Welsbie et al., 2013). Finally, hiw and phrl mutants maintain synaptic connections and block axon loss in Drosophila and mouse models of Wallerian degeneration (Massaro et al., 2009; Xiong et al., 2012; Babetto et al., 2013). The vital role of axonal and synaptic maintenance in circuit formation and neurodegenerative diseases underscores the importance of understanding how these processes are regulated, and suggests that the DLK pathway may be a therapeutic target. 
The Wnd/DLK pathway can be potentiated by mechanisms that affect either Wnd/DLK activation or levels (Yan and Jin, 2012; Huntwork-Rodriguez et al., 2013; Valakh et al., 2013). Two known mechanisms that lead to an increase of Wnd protein levels are loss of the E3 ubiquitin ligase Hiw and overexpression of the deubiquitinating protease Fat Facets (Faf). Similar to hiw mutants, neuronal overexpression of Faf leads to increased Wnd levels and synaptic overgrowth (Collins et al., 2006). Combining these two negative regulators results in synthetic lethality due to increased activation of the pathway (DiAntonio et al., 2001). Therefore, we hypothesized that we may identify additional negative regulators by screening for mutants that are lethal in combination with neuronal overexpression of Faf. Combining the power of Drosophila genetics with whole genome sequencing, we have identified SkpA as a novel negative regulator of the DLK-Jnk pathway.

Skp proteins are core components of SCF-type E3 Ubiquitin ligases. SCF complexes are multicomponent E3 ubiquitin ligases that include a member from each of the Cullin, Skp1, Rbx1 (ring domain), and F-Box protein families. Hiw appears to function as a component of an SCF-like Ubiquitin ligase complex (Liao et al., 2004; Saiga et al., 2009) with Hiw serving as a novel ring domain protein. In Drosophila, there are six Skp1 proteins (A-F) with SkpA being the most widely expressed (Murphy, 2003). Here we identify SkpA as an essential component of the Hiw complex and demonstrate a role for SkpA in both synaptic development and axonal maintenance.

\section{Materials and Methods}

Fly stocks. Drosophila melanogaster were raised on standard fly food at $25^{\circ} \mathrm{C}$. The following strains were used in this study: Canton $\mathrm{S}$ or $\mathrm{w}^{-}$(wild-type), $w n d^{1}$ and $w n d^{2}$ (Collins et al., 2006), Run ${ }^{3}$, and FM7TW9 (Bloomington Stock Center), elav Gal4 (neuron-specific; Yao and White, 1994), $S k p A^{1}$ (Murphy, 2003), $S k p A^{60389}$ and $S k p A^{d f 9053}$ (Bloomington Stock Center), G7-Gal4 (muscle-specific; Zhang et al., 2001), m12-Gal4 (single-axon resolution; Ritzenthaler et al., 2000), OK6Gal4 (motoneuron-specific; Aberle et al., 2002), UAS-jun ${ }^{D N}$ and UAS$f o s^{D N}$ (Eresh et al., 1997), UAS-bsk ${ }^{D N}$ (Weber et al., 2000), hiw ${ }^{\Delta N}$ (Wu et al., 2005), hiw ${ }^{N D 51}$ (Wan et al., 2000), and puc-LacZ ${ }^{\mathrm{E} 69}$ (Martín-Blanco et al., 1998). RNAi lines used from the Vienna Drosophila RNAi Consortium ( $\alpha$-Spectrin, 110417 and $S k p A, 107815)$ and Bloomington Trip lines (Nmnat, 29402). Generation of UAS-Fat Facets was described previously (DiAntonio et al., 2001). Clone UFO01602 was obtained from DGRC for generation of UAS-SkpA-Flag-HA that was used in rescue experiments. The UAS-SkpA-Flag-HA transgene was inserted into the attP2 landing site ( $\sim 68 \mathrm{~A} 4$; Bloomington stock no. 8622 ) by Best Gene. Male and female larvae were used for experiments unless stated.

Synthethic lethality screen. Isogenic $\mathrm{w}^{-}$males were fed a solution of $5 \%$ sucrose and $14 \mathrm{~mm}$ methanesulfonic acid ethyl ester (EMS) for $24 \mathrm{~h}$. These males were crossed to $\mathrm{run}^{3}$ /FM7TW9 virgin females, and $\sim 7000$ $\mathrm{w}^{-},{ }^{-{ }^{2} S^{*}}$ /FM7TW9 virgin females were collected. These females were mated singly to $\mathrm{w}^{-} / \mathrm{y}$; elav-Gal4 $\left(\mathrm{w}^{+}\right), E P(3) 381-F a f\left(w^{+}\right) / \mathrm{TM} 6 \mathrm{~B}$ males. The progeny from the individual crosses were scored for sex and eye color through the side of the vial to rapidly determine genotype and identify whether the EMS mutant is (1) lethal on its own, (2) lethal in combination with neuronal expression of Faf, or (3) not lethal. Six synthetic lethal mutants were obtained that failed to complement a mutant hiw allele.

Mapping SkpA. The GD65 locus was mapped by meiotic recombination and deficiency mapping to 1B14-1E1. Whole-genome sequencing identified a lone missense mutation in this region that corresponded to the $S k p A$ gene. Additional $S k p A$ mutant alleles complemented the GD65 mutant phenotype suggesting that GD65 was allelic to $S k p A$.

Whole-genome sequencing. DNA for sequencing was prepared from $>35$ female wandering third instar larva that were homozygous for the GD65 mutation, $\mathrm{w}^{-}$control line, and an additional GD control line. Larva were briefly rinsed in $50 \%$ bleach solution to remove surface con- tamination and frozen at $-80^{\circ} \mathrm{C}$ for at least $1 \mathrm{~h}$. Larvae were crushed with a small pestle and genomic DNA was prepared using the DNeasy Blood and Tissue Kit protocol. Three micrograms of genomic DNA was used for library construction. Genomic DNA was sonicated to an average size of 175bp. The DNA fragments were repaired to produce blunt ends then modified with a 3' "A" base overhang and ligated to Illumina's standard sequencing adapters. The ligated fragments were amplified for 10 cycles incorporating a unique indexing sequence tag. The resulting libraries were sequenced as 101-nucleotide paired-end reads using the Illumina HiSeq-2000. More than 30 million reads resulting in 13.8 million aligned read pairs were generated for GD65. This resulted in $24.2 \times$ coverage of the genome (control lines: $34.4 \times$ coverage for the $\mathrm{w}^{-}$line and $30.2 \times$ coverage for the additional GD line). Data analysis was done through GTAC: reads were aligned to the genome using Novoalign; SNPs identified using SAMtools and annotated using snpEFF. Comparison of the exons of the X-chromosome of GD65 mutant strain with control lines identified 298 unique nonsynonymous mutations. Library construction and sequencing was performed at GTAC at Washington University School of Medicine.

Immunocytochemistry. Larval filet preps were fixed in Bouin's solution (a 1:5:15 ratio of acetic acid/formalin/picric acid) for $10 \mathrm{~min}$ at room temperature unless otherwise noted. Blocking and staining were performed in PBS $+0.1 \%$ Triton X-100 $+5 \%$ goat serum The rabbit $\alpha$-DVGLUT antibody was described previously (Daniels et al., 2004), and used at 1:5000 dilution. Other antibodies used were as follows: Cy3 and Cy5-conjugated goat $\alpha$-HRP (1:1000, Jackson ImmunoResearch), mouse $\alpha$-LacZ (1:100, 40-1a; fix in 4\% paraformaldehyde in PBS on ice for 40 min; Developmental Studies Hybridoma Bank), mouse $\alpha$-DLG monoclonal antibody 4f3 (1:2000, developed by Corey Goodman, Renovis). AlexaFluor 488-conjugated $\alpha$-rabbit (1:1000, Invitrogen) and Cy3 $\alpha$-mouse (1:1000, Jackson ImmunoResearch) were used at 1:1000 dilution. All samples were mounted and imaged in $70 \%$ glycerol containing Vectashield.

Nerve crush assay. The segmental nerves of third instar larvae were visualized through the cuticle under a dissection microscope. Larvae were immobilized in a cold dish and the segmental nerves were pinched tightly through the cuticle for $5 \mathrm{~s}$ with Dumostar no. 5 forceps. After injury, the larva were transferred to a grape plate and kept alive for varying periods of time at $25^{\circ} \mathrm{C}$ before dissection. This assay was adapted from (Xiong et al., 2010). Synapses were stained with $\alpha$ DVGLUT and $\alpha$ HRP. Axons were labeled with GFP expressed by the M12-Gal4 driver. GFP stained larval filet preps were fixed in PBS $+4 \%$ paraformaldehyde for $20 \mathrm{~min}$ at room temperature.

Imaging and analysis. Images of Drosophila third instar larva were acquired on a Nikon Eclipse C1 Confocal microscope using $40 \times$ or $60 \times$ oil objectives. Images shown are maximal Z-projections of confocal stacks. Samples for each experiment were processed simultaneously and used identical confocal gain settings. MetaMorph software (Molecular Devices) was used for bouton size, synaptic area, and intensity measurements. Synaptic retractions were counted as a percentage of NMJs at segments A3-A6 of muscle 6/7 NMJs where postsynaptic Dlg staining is not apposed by presynaptic VGLUT staining. The mean intensity of LacZ expression was quantified in MetaMorph by measuring the intensity of LacZ staining in the neuronal nuclei that lie along the dorsal midline of the nerve cord. The mean lacZ intensity per nucleus was measured for each genotype and normalized against wild-type controls.

Tandem-affinity purification procedure. Purification of the Hiwassociated protein complex was previously described (Wu et al., 2007; Tian et al., 2011). To purify the DFsn-associated protein complex, a UAS-TAP-DFsn transgene was generated using a full-length DFsn cDNA (Wu et al., 2007), and was expressed in fly heads by the BG380-Gal4 driver. Tandem affinity purification (TAP) purification was performed as previously described (Tian et al., 2013). Mascot scores $>36=p<$ 0.05 .

Electrophysiology. Intracellular electrophysiological recordings were done as previously reported (Marrus and DiAntonio, 2004). Briefly, wandering third instar larvae were dissected in $0.42 \mathrm{mM} \mathrm{Ca}^{2+}$ Stewart saline, HL3; Stewart et al., 1994). Both spontaneous miniature excitatory junction potentials (mEJPs) and evoked EJPs were then recorded in 0.42 
mM Ca ${ }^{2+} \mathrm{HL} 3$ from muscle 6 in segments A2 and A3. Seventy-five consecutive miniature events were measured per cell using MiniAnalysis (Synaptosoft) and averaged to determine mean mEJP. Events with a slow rise time were rejected as artifacts from neighboring electrically coupled muscle cells. To record evoked EJPs, segmental nerves were cut and suctioned into a stimulating electrode, where they received a brief ( $1 \mathrm{~ms}$ ) depolarizing pulse. The amplitude of the stimulus was set to ensure recruitment of both nerves innervating muscle 6. EJP amplitude was determined by averaging 75 consecutive EJPs delivered at $0.2 \mathrm{~Hz}$ using Clampfit 9.0. Quantal content was estimated by dividing the mean EJP by the mean $\mathrm{mEJP}$. All cells displayed input resistances $>5 \mathrm{M} \Omega$ and resting potentials of $-60 \mathrm{mV}$ or lower. Statistical analysis was performed using a $t$ test to compare pairs of samples or ANOVA for more than two samples in an experimental group.

\section{Results}

A genetic screen for modulators of the

Hiw-Wnd-Jnk pathway

The Hiw-Wnd-Jnk pathway is a central player in multiple aspects of axonal and synaptic biology. The finding that Hiw and DLK/ Wnd function not only in synaptic development, but also in response to injury, has raised the possibility that this pathway is a potential target for therapeutic intervention. To identify novel genes that regulate the Wnd pathway, we have performed a forward genetic screen for new modulators of the pathway. The screen takes advantage of the previous observation that hiw mutants are lethal in combination with neuronal overexpression of the ubiquitin hydrolase Fat Facets (Faf; DiAntonio et al., 2001). Similar to hiw mutants, overexpression of Faf leads to an increase in Wnd levels and an expansion of the NMJ (DiAntonio et al., 2001; Collins et al., 2006). The synthetic lethality of hiw mutants and Faf overexpression is likely due to excessive Wnd signaling, since removing one copy of wnd from this genetic background allows the animals to survive (Collins et al., 2006). We reasoned, therefore, that additional mutants that were lethal with neuronal overexpression of Faf may also be negative regulators of Wnd.

The screen is depicted in Figure $1 A$. Mutant females were individually crossed to flies that neuronally overexpress Faf and which are marked by a transgene that results in red eye color. The ability to track genotype by eye color allows for rapid identification of EMS mutants that are (1) lethal, (2) lethal in combination with neuronal expression of Faf, or (3) not lethal. Mutants that are lethal only in combination with neuronal overexpression of Faf are candidate modulators of the Hiw-Wnd-Jnk pathway. We screened $\sim 5500$ flies and identified 20 mutants that were lethal with Faf overexpression. Fourteen of the mutations mapped to hiw, which was expected because hiw is on the X-chromosome and is a giant target for mutagenesis due to its large size (5233 aa). However, the remaining alleles are potential novel regulators of the Hiw-Wnd pathway.

Because Faf may have targets in addition to Wnd, we performed a secondary screen to identify mutants whose synthetic lethality with overexpressed Fat Facets is suppressed by wnd mutants to focus on negative regulators of the Wnd pathway. Of the six mutants, two nonallelic mutants were suppressed by loss of function mutations in wnd, including an allele we term GD65. GD65 was crossed to a strain that neuronally expresses Faf and the resulting progeny from the cross were counted. No GD65 mutant progeny expressing Faf were observed, whereas 146 GD65 mutant progeny containing the balancer chromosome were observed (Fig. 1Bi). This lethality was suppressed by a wnd mutation as demonstrated by viability of the triple mutant (24 progeny vs $\sim 40$ expected progeny; Fig. 1Bii). Because GD65 is likely func-

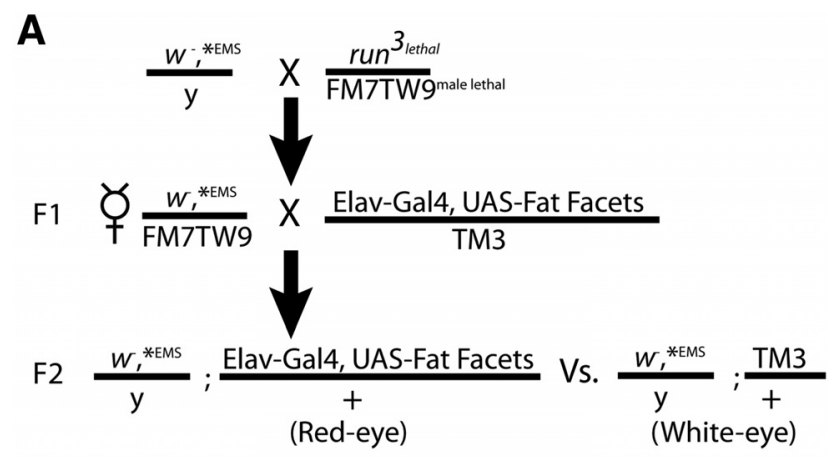

\section{B Wallenda mutants suppress the lethality of GD65 and neuronal expression of Fat Facets}

i. GD65/y; UAS-Faf, elav-Gal4/+
GD65/y; TM3/+
ii. GD65; wnd $^{-1}$, UAS-Faf/wnd ${ }^{-2}$, elav-Gal4
GD65; wnd $^{-1}$, UAS-Faf/TM6B
GD65; wnd $^{-2}$, elav-Gal4/TM3

offspring \#

0 146

Figure 1. Identification of genes involved in the Hiw-Wnd pathway. A, Scheme for isolating $X$-chromosome mutations that are synthetic lethal with neuronal overexpression of Faf is shown. EMS-treated $w^{-}$males were mated to run $^{3} /$ FM7TW9 (male lethal) virgin females in mass to generate mutagenized $F 1$ females. $F 1$ virgin females were individually crossed to males neuronally expressing Faf. Mutant $\mathrm{F} 2$ males were scored for lethality with overexpression of Faf based on eye color that is induced by the presence or absence of the transgene marked by the mini $\mathrm{w}^{+}$gene. $\boldsymbol{B}$, Quantification of lethality and wnd suppression. $\mathbf{B i}, G D 65$ is lethal with neuronal overexpression of Faf. GD65 mutants were crossed to UAS-Faf, elav-Gal4/TM3 and the resulting male offspring were scored. Bii, wnd mutants suppress synthetic lethality. GD65; wnd ${ }^{7}$, UAS-Faf/TM3 females were crosses to GD65; wnd ${ }^{2}$, elav-Gal4/TM6B males. Male offspring from the cross were scored demonstrating wnd suppression of the synthetic lethality.

tioning as a modulator of the Wnd pathway, we focused on the identification and characterization of this mutant.

\section{GD65 acts to restrain synaptic growth}

Because increased Wnd levels can lead to NMJ overgrowth, we reasoned that if GD65 is a negative regulator of Wnd, then it may show an increase in synaptic bouton number. Therefore, we characterized the synaptic morphology of GD65 mutants at the NMJ of muscle 4, which is formed from a single motoneuron (MN4b), but similar results were observed at all glutamatergic type I NMJs. GD65 mutants displayed a more than twofold increase in synaptic bouton number and synaptic branch number with a concurrent $60 \%$ decrease in bouton size (Fig. $2 A$ ). The synaptic phenotypes of GD65 are similar to hiw mutants although quantitatively less pronounced (Wan et al., 2000).

Interestingly, GD65 mutant phenotypes are strongly affected by maternal genotype. Significant overgrowth of the NMJ is seen only when mothers are homozygous for the GD65 mutation (Fig. $2 B$ ). Homozygous GD65 mutant progeny from heterozygous mutant mothers have an $82 \%$ decrease in synaptic terminal overgrowth compared with those from homozygous mutant mothers (Fig. $2 B$ ). In addition, heterozygous progeny from homozygous mutant mothers have a $60 \%$ increase in bouton number compared with heterozygous progeny from heterozygous mothers (Fig. 2B). Therefore, the maternal contribution of the GD65 gene product is essential for proper synaptic terminal development.

\section{Identification of GD65 as $S k p A$}

Because GD65 genetically interacts with wnd mutants and regulates synaptic development, we chose to identify the mutated 
A
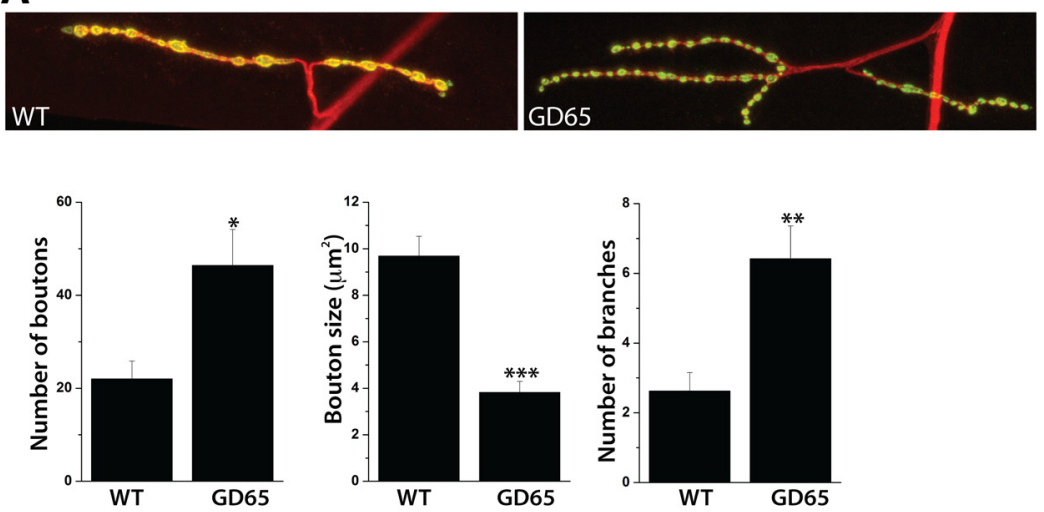

B

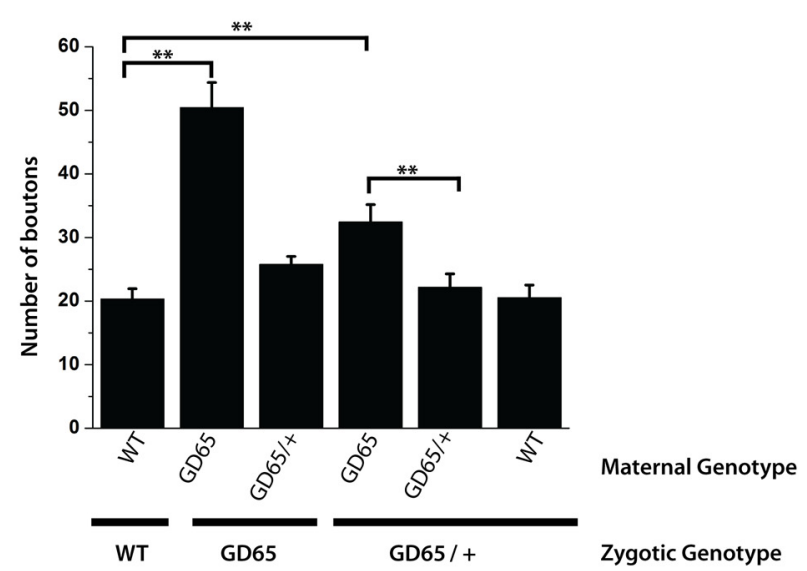

Figure 2. GD65 restrains synaptic growth at the larval NMJ. $\boldsymbol{A}$, Representative confocal images of muscle 4 NMJ synapses demonstrating a large increase in synaptic branches and synaptic boutons in GD65 mutants (axons in red, $\alpha$-HRP; boutons green, $\alpha$-DVGLUT). The graphs show quantification of bouton number ( $\left.22 \mathrm{vs} 46,{ }^{*} p<0.05\right)$, bouton size $\left(9.68\right.$ vs $\left.3.82 \mu \mathrm{m}^{2}\right)$, ${ }^{* * *} p<$ 0.001 ), and branch number ( 2.6 vs $6.4,{ }^{* *} p<0.01$ ) for WT and the GD65 mutants ( $n=12$ ). $\boldsymbol{B}, G D 65$ mutants exhibit a strong maternal effect on synaptic growth. Maternal and zygotic genotypes are listed for each genotype tested. Quantification of bouton number from motoneuron MN4b on muscle 4 is shown. Bouton number $=20.3,50.4,25.8,32.4,22.1,20.5$, and $N=16,15,15$, $13,16,14$ respectively for each genotype. Error bars indicate $\pm S E M ;{ }^{* *} p<0.01$.

gene. To this end, we used a combination of molecular mapping techniques and next generation whole genome sequencing of the GD65 mutant strain. DNA libraries were created for whole genome sequencing from homozygous GD65 larvae as well as two control lines (the wild-type line that was used for the EMS screen and a different GD line that originated from the screen). Because GD65 is on the X-chromosome, we focused on mutations that were unique to the X-chromosome of GD65. Comparing the $\mathrm{X}$-chromosome sequence of the GD65 line against the wild-type and sister GD line allowed us to identify 298 unique nonsynonymous mutations in the exons of the X-chromosome. Not all of the 298 mutations were likely due to the EMS mutagenesis, but may be due to additional polymorphisms that existed on the chromosomes sequenced.

To define the region of the X-chromosome carrying the mutation, we used recombination and deficiency mapping techniques. Results from recombination mapping for GD65 indicated that the mutation was located on the left side of the X-chromosome allowing us to focus our deficiency mapping on location 1A-3A of the chromosome. Deficiencies were crossed to GD65 and the transheterozygous mutant was tested for synthetic lethality with Faf overexpression. One deficiency recapitulated the GD65 lethality phenotype and was deleted for region 1B14; 1E1 and contained 47 genes. Upon analyzing our whole genome sequencing data for this region, there was only a single gene, $S k p A$, which contained a mutation that altered a coding sequence in the region deleted by the deficiency.

To explore whether the GD65 phenotype was indeed due to the mutation in the SkpA locus, we performed genetic complementation tests with additional alleles of $S k p A$. $S k p A$ is an essential gene and null alleles are lethal (Murphy, 2003). Therefore, to test for complementation of GD65 we took advantage of the maternal effect of GD65. Heterozygous $S k p A$ mutant females were crossed to GD65 males to produce $S k p A / G D 65$ females. Only if $S k p A$ complements the GD65 mutation will male offspring (GD65/y) of this cross exhibit synaptic overgrowth because mothers must be homozygous for GD65 to exhibit a strong phenotype. To test for complementation, we used the $S k p A^{1-n u l l}$ allele produced by imprecise excision, (Murphy, 2003), a P-element insertion $S k p A^{60389}$, and a deficiency of the region (df9053). As shown in Figure 3, offspring from these GD65/Skp mothers exhibit a similar, but quantitatively stronger, NMJ overgrowth phenotype than offspring from GD65 homozygous mothers. Therefore, GD65 is allelic to $S k p A$. Because the GD65 allele is viable and homozygous GD65 mothers produce offspring with a weaker synaptic phenotype than do $S k p A^{1} / G D 65$ mothers, GD65 is a hypomorphic allele of $S k p A$. We named this new allele $S k p A^{G D 65}$.

We resequenced the $S k p A$ gene and confirmed the mutation identified by whole genome sequencing. The mutation $\mathrm{G}_{94} \mathrm{~A}$ results in a glutamic acid to lysine substitution altering the charge of the amino acid. Based on the Skp1 protein structure (Schulman et al., 2000; Zheng et al., 2002), this mutation maps to helix 2 of SkpA. This mutation may partially disrupt the stability or function of the protein or may partially disrupt the ability of F-Box proteins to interact with SkpA because it is near the F-Box binding domain. Indeed, mutations near the F-Box binding domain in Skp1 have the ability to partially disrupt SCF-complex function (Brace et al., 2006).

As an independent test of whether GD65 is allelic to $\operatorname{Skp} A$, we performed rescue experiments with $S k p A$. $S k p A^{1} / S k p A^{G D 65}$ females containing a UAS-SkpA transgene were crossed to various GAL4 drivers and tested for rescue of the synaptic overgrowth phenotype. Neuronal expression, but not muscle expression, of $S k p A$ completely rescued the zygotic phenotype of $S k p A^{G D 65}$ mutants (Fig. $4 A$ ). This result not only confirms that GD65 is indeed allelic to $\operatorname{Skp} A$, but demonstrates that $S k p A$ functions in the neuron to regulate synaptic growth. Neuronal expression of RNAi targeting $S k p A$ also leads to synaptic overgrowth, confirming the neuronal role of the protein and demonstrating that the phenotype is due to reduced function of SkpA rather than to any peculiarity of the GD65 allele (Fig. 4B). Additionally, knockdown of SkpA selectively in motoneurons with OK6-Gal4 also led to overgrowth of the NMJ (WT = 22.4 boutons; SkpARNAi $=38.7 ; p>0.001$ ), indicating a cell autonomous requirement for SkpA within the motoneuron. 

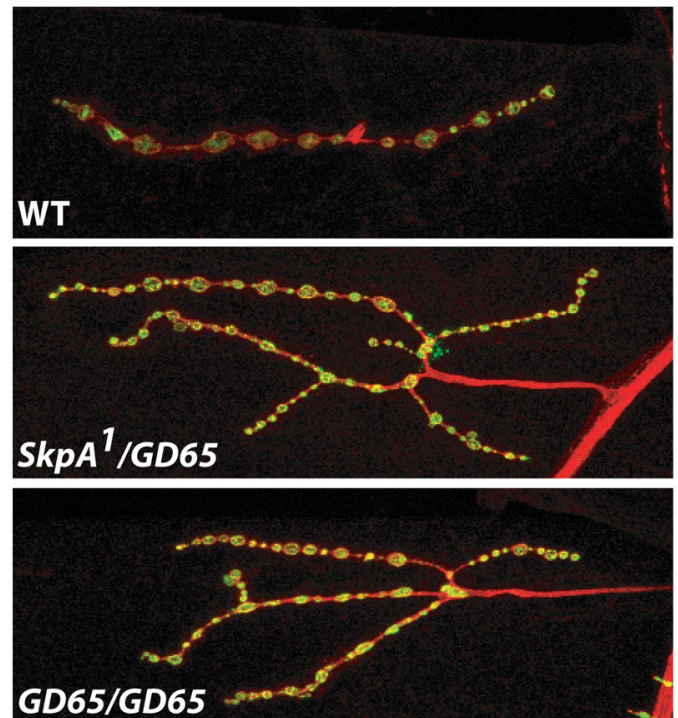

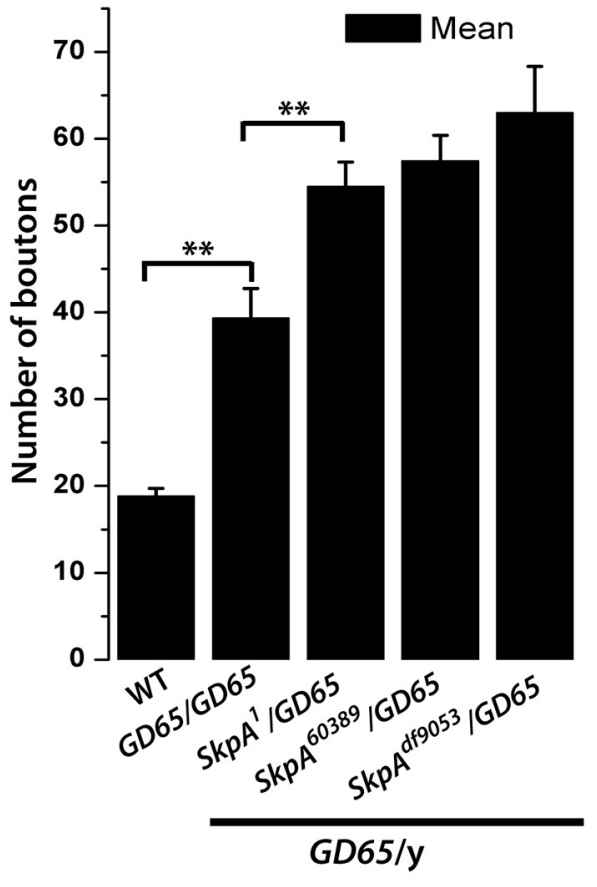

maternal genotype

\section{Zygotic genotype}

Figure 3. GD65 is identified as SkpA. Representative confocal images from muscle 4 NMJ synapses of third instar larva (axons in red, $\alpha$-HRP; boutons green, $\alpha$-DVGLUT). Multiple alleles of SkpA recapitulate the GD65 phenotype. Quantification of bouton number: WT (18.8), GD65/GD65 (39.3), SkpA /GD65 (54.5), SkpA $A^{60389} / G D 65$ (57.4), and SkpA ${ }^{d f 9053} / G D 65$ (63); N = 22, 19, 23, 14, 13, respectively. Error bars indicate \pm SEM; ** $p<0.01$.

\section{SkpA genetically interacts with hiw}

Hiw and its homologues likely function as part of an SCF-like Ubiquitin ligase complex to regulate synaptic growth as both Hiw and the F-Box protein Fsn play a role in regulating synaptic growth (Liao et al., 2004; Wu et al., 2007; Saiga et al., 2009). In C. elegans, the Skp homolog Skr-1 physically interacts with Rpm-1 and Fsn-1 (Liao et al., 2004), but no functional data exists demonstrating that Skr-1 participates in the Rpm-1 ligase complex that functions in regulating neuronal development.

Our characterization of SkpA suggests that SkpA likely functions with Hiw to restrain synaptic growth. If so, then our hypomorphic allele $S k p A^{G D 65}$ should enhance the phenotype of an allele of hiw that has an intermediate phenotype, hiw ${ }^{N D 51}$. Individually, both hypomorphic alleles exhibit a modest increase in bouton number at muscle $4\left(\mathrm{WT}=18.3 ; \operatorname{Skp} A^{G D 65}=40.4\right.$; $h i w^{N D 51}=35.8$; Fig. $5 A$ ). The synaptic overgrowth phenotype is dramatically enhanced in the double mutant $\left(\operatorname{Skp} A^{\mathrm{GD} 65}\right.$, $h i w^{N D 51}=91$ ), suggesting that SkpA and Hiw function in the same pathway. Consistent with this hypothesis, this double mutant has a phenotype similar to that of a hiw-null $\left(h i w^{\Delta N}=\right.$ 110; Fig. 5A).

\section{SkpA functions with Hiw to regulate synaptic function}

Both hiw and $D f_{s} n$ mutants have defects in synaptic function with decreases in EJP amplitude and quantal content (Wan et al., 2000; Wu et al., 2007). Although the morphological phenotype of hiw is due to activation of the Wnd pathway, the physiology defects are independent of Wnd as mutants in Wnd do not suppress the functional defect (Collins et al., 2006). Because other Skp proteins may be functioning with Hiw to regulate synaptic release, we wanted to test whether SkpA also affected synaptic function. Although there is a trend toward a decrease in EJP amplitude and quantal content in the $S k p A$ mutant, it is not statistically signif- icant, potentially due to the hypomorphic nature of the $\operatorname{Skp} A^{G D 65}$ allele (Fig. 5B).

As a second test of the role of SkpA in regulating synaptic transmission, we combined a hypomorphic allele of hiw with the $S k p A^{G D 65}$ mutant allele. The hypomorphic hiw ${ }^{N D 51}$ allele also has no statistically significant effect on EJP amplitude or quantal content (Fig. 5C). This is not surprising because we previously demonstrated that the synaptic growth phenotype of hiw mutants is more sensitive to loss of Hiw function than is the synaptic function phenotype (Wu et al., 2005). In contrast to the single mutants, the combination of the two hypomorphic alleles leads to a pronounced defect in evoked release, with a decrease in EJP amplitude (69\% decrease) and quantal content (69\% decrease; Fig. $5 C)$. There is no significant difference in mEJP amplitude in any of the mutants $\left(\mathrm{WT}=0.90 \pm 0.04 \mathrm{mV}\right.$; hiw ${ }^{N D 51}=0.89 \pm 0.05$ $\mathrm{mV} ; S k p A^{G D 65}=0.85 \pm 0.02 \mathrm{mV}$; and hiw ${ }^{N D 51}, S k p A^{G D 65}=$ $0.92 \pm 0.07 \mathrm{mV}$ ). Hence, SkpA does regulate synaptic function, and likely works together with Highwire in this process.

\section{SkpA interacts with Hiw and DFsn}

We used a proteomics approach to test for a physical interaction between SkpA and other components of the Highwire complex. Our prior work demonstrated that Highwire binds to the F-box protein DFsn and that these proteins function together in a complex (Wu et al., 2007). We purified the Hiw-associated protein complex (Tian et al., 2011) and the DFsn-associated protein complex from Drosophila adult brains using a transgene-based two-step purification method, TAP (Rigaut et al., 1999; Tian et al., 2013). Proteins present in either the Hiw-associated or DFsnassociated complex were identified via liquid chromatographytandem mass spectrometry (LC-MS/MS). DFsn was identified in the Hiw-associated protein complex (Wu et al., 2007), together with three peptides of SkpA: VDQGTLFELILAANYLDIK, GL- 


\section{A}
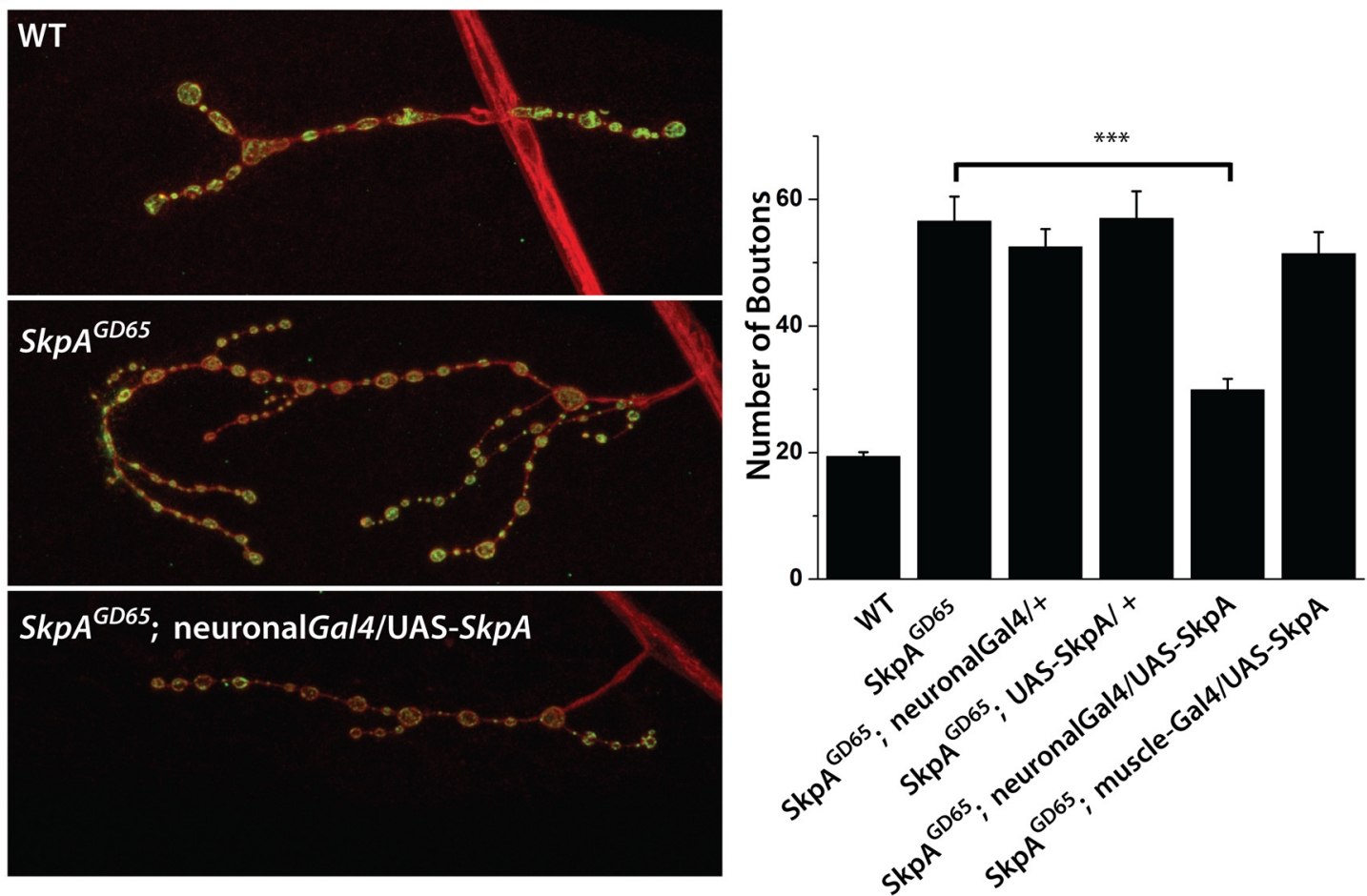

B
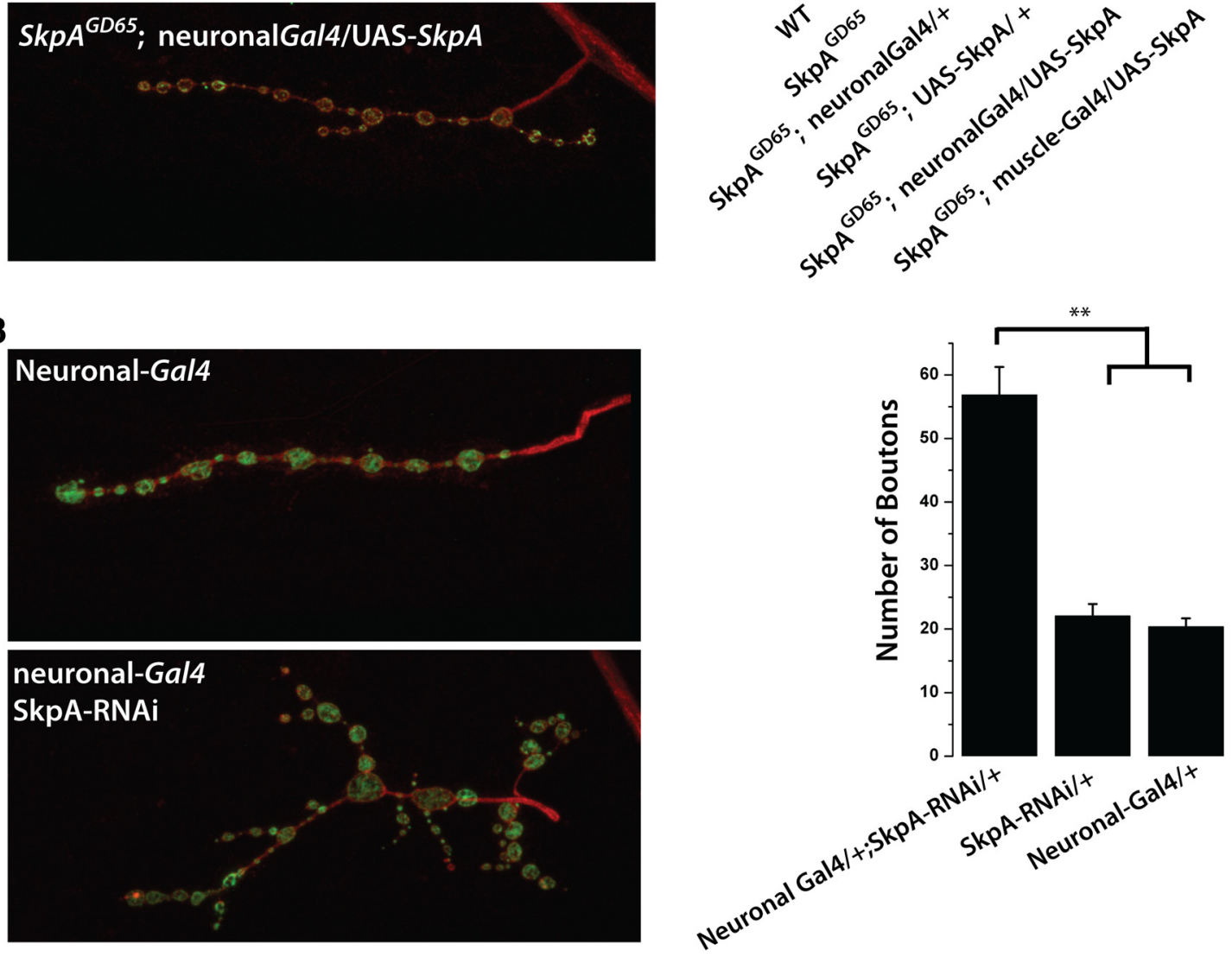

Figure 4. SkpA functions in the motoneuron to regulate synapse morphology. A, Neuronal expression of SkpA rescues the synaptic morphology phenotype of $S k p A^{6 D 65}$. Representative confocal images of muscle 4 synapses from third instar larva costained with DVGLUT (green) and HRP (red). Genotypes of the progeny are listed in figure. Elav-Gal4 was used for neuronal expression. (Note: the maternal genotypes for the $S \mathrm{kpA}$ progeny were $S \mathrm{kpA} \mathrm{A}^{7} / \mathrm{SkpA^{GD65 }}$ ). The graph depicts quantification of bouton number from motoneuron MN4b on muscle 4. The morphological defects in $S \mathrm{kp} \mathrm{A}^{\mathrm{GD65}}$ are rescued by presynaptic expression of UAS-SkpA (*** $<0.001$ for $S k p A A^{G D 65}{ }_{v S S k p A}{ }^{G D 65}$; elavGal4/UAS-SkpA). $N=48,25,36,20,27$, and 26, respectively, for each genotype. Error bars indicate \pm SEM. $\boldsymbol{B}$, Neuronal knockdown of SkPA leads to overgrowth at the NMJ. Elav-Gal4 was used for neuronal expression of the RNAi (UAS-SkpARNAi ${ }^{107815}$ ). Images and quantification are the same as above $\left.{ }^{* *} p<0.01\right) . N=22,17$, and 19 , respectively, for each genotype.

LELTCK, and TPEEIRK (Mascot score: 36, 52, 27; accumulating Mascot score: 115). In the DFsn-associated protein complex, Hiw was identified as the top DFsn-interacting protein due to its large molecular weight. In addition, four peptides of SkpA were also identified: GLLELTCK, KENEWCEEK, DFSPAEEEQVR, and KDFSPAEEEQVRK (mascot score: 55, 54, 66, and 69; accumulating Mascot score: 244$)$. Other Skp proteins, such as SkpB and $\mathrm{SkpC}$, were not identified in either protein complex suggesting that the Hiw complex may be specific to SkpA, although other Skp and SCF components can function at the synapse (Juo and Kaplan, 2004). These biochemical data in conjunction with the genetic data above strongly support the model of a Hiw/DFsn/SkpA protein complex in Drosophila neurons.

SkpA signals through the MAPKKK Wallenda to regulate synaptic growth

How does SkpA regulate synaptic growth? The screen was designed to identify new negative regulators of the Wnd path- 
way. If $\operatorname{skpA}$ were a new negative regulator of Wnd, we would predict that wnd mutants should suppress the NMJ overgrowth phenotype of $S k p A \mathrm{mu}-$ tants. We tested whether the overgrowth of $S k p A$ mutants requires Wnd function. As shown in Figure $6 A$, wnd mutants can significantly suppress the increase in bouton number in $S k p A$ mutants, demonstrating that Wnd is necessary for synaptic overgrowth in $S k p A$ mutants. Interestingly, $S k p A$ mutants are not completely suppressed by mutations in wnd in contrast to the full suppression of mutations in hiw and Dfsn by wnd (Collins et al., 2006; Wu et al., 2007). These results are consistent with SkpA, as part of non-Hiw SCF ligase complexes, targeting additional unknown substrates that account for the small increase in bouton number that is not suppressed by a wnd mutant.

\section{Jnk signaling is increased in SkpA mutants}

Because Wnd/DLK signals through Jnk, we tested whether the Jnk pathway is upregulated in $S k p A$ mutants. Jnk positively regulates expression of the Puckered gene and therefore expression of Puckered is an indicator of Jnk activity. As a readout of Puckered expression we used a LacZ enhancer trap under the control of the endogenous Puckered promoter (MartínBlanco et al., 1998). We measured the intensity of LacZ staining in neuronal cell bodies in third instar larva. Compared with wild-type larva, $S k p A$ mutants exhibited an $\sim 90 \%$ increase in LacZ staining intensity (Fig. 6B).

Because Jnk signaling is elevated in $S k p A$ mutants we tested whether this increase is responsible for the overgrowth phenotype. We expressed a dominantnegative form of Jnk, containing a nonfunctional kinase domain (Weber et al., 2000), to inhibit Jnk signaling in $S k p A$ mutants. Neuronal expression of Jnk ${ }^{\mathrm{DN}}$ in a $S k p A$ mutant partially suppresses the SkpA mutant phenotype (Fig. 6C), consistent with a role for Jnk downstream of SkpA in regulating synaptic overgrowth.

Jnk often signals through the AP-1 transcription factors Fos and Jun. Fos and Jun have previously been linked to synaptic growth (Sanyal et al., 2003) and we have shown that Fos functions downstream of Hiw to regulate synaptic growth (Collins et al., 2006). To test whether Fos and/or Jun functions in SkpA regulated synaptic growth, we neuronally expressed dominant negative forms of each protein in $S k p A$ mutants. Expression of both Fos ${ }^{D N}$ and $J u n^{D N}$ suppresses the overgrowth of $S k p A$ mutants consistent with SkpA functioning through AP-1 (Fig. 6C). We had not previously observed suppression of hiw-null mutants
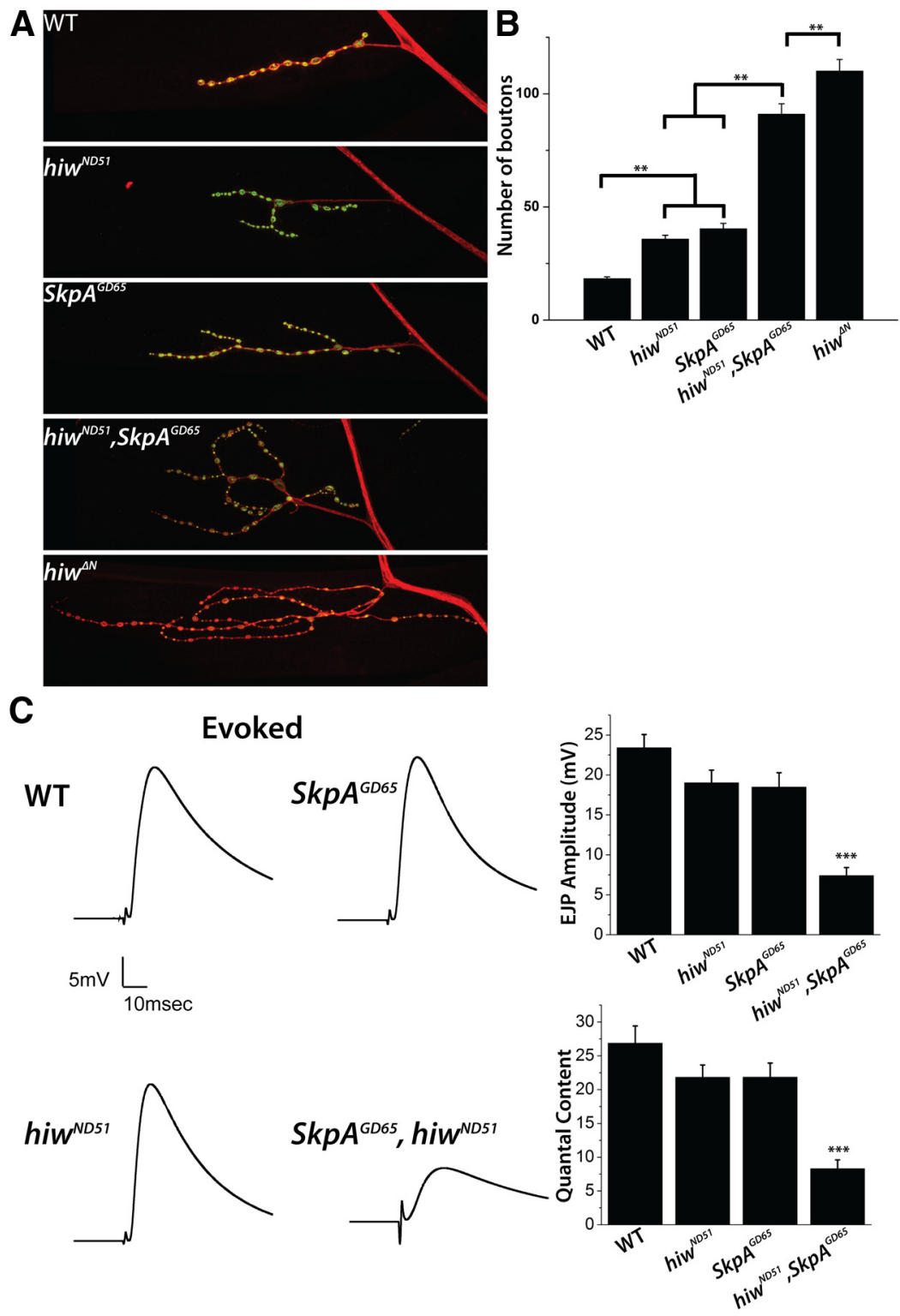

Figure 5. SkpA genetically interacts with hiw to restrain synaptic growth. $\boldsymbol{A}$, Representative confocal images of muscle 4 synapses from third instar larva costained for presynaptic DVGLUT (green) and neuronal HRP (Red). Genotypes of the progeny are listed in the figure. In each case, the maternal genotype is identical to the genotype of progeny. $\boldsymbol{B}$, Quantification of bouton number from motoneuron MN4b on muscle $4(18.3,35.8,40.4,91.0,110.0$, respectively). There is a significant increase $\left({ }^{* *} p<0.01\right)$ in the number of boutons between WT and all genotypes. There is also a significant increase between the single-mutants and the double-mutant $\left(S \mathrm{KpA}^{6 D 65}\right.$, hiw $\left.{ }^{N D 51}\right)$ demonstrating an enhancement of the phenotype. $N=30,33,33,28,28$. C, Evoked EJP and quantification of EJP amplitude and quantal content from muscle 6 of third instar larva for each of the genotypes listed. There is a significant decrease $\left(^{* * *} p<0.001\right)$ in EJP amplitude and quantal content of $S \mathrm{kp} \mathrm{A}^{G D 65}$, hiw ${ }^{N D 51}$ and each of the other genotypes.

by $J u n^{D N}$, likely because the hiw mutants have a stronger phenotype than the hypomorphic $S k p A^{G D 65}$ allele.

\section{Mutants in $S k p A$ protect synapses and axons from degeneration after injury}

The findings above are consistent with the model that SkpA is an essential component of the Hiw/E3 Ub ligase complex that restrains NMJ expansion. Because there are six genes encoding Skp proteins in Drosophila, we wished to assess whether SkpA participates in other Hiw-dependent processes. Hiw regulates synaptic stability in Drosophila as hiw mutants suppress the synaptic loss induced by $\alpha$-spectrin mutants in a Drosophila model of motor neuron disease (Massaro et al., 2009). 
A
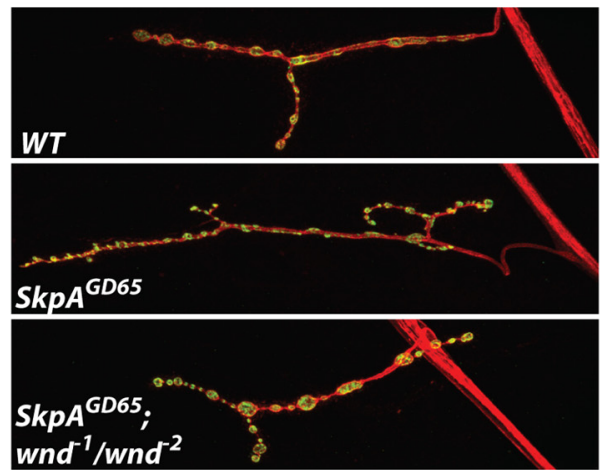

wnd $^{-1} /$ wnd ${ }^{-2}$

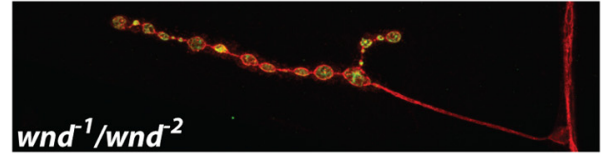

B
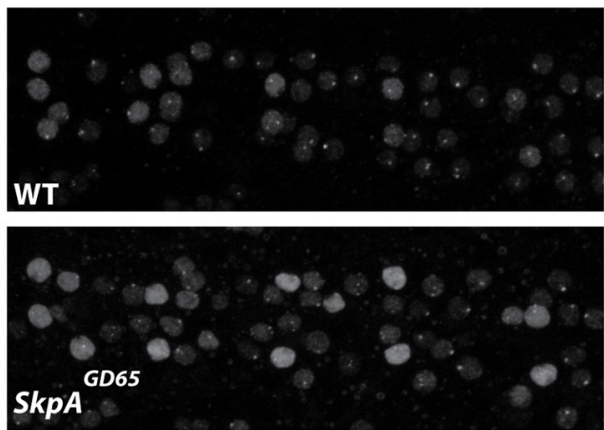

C WT
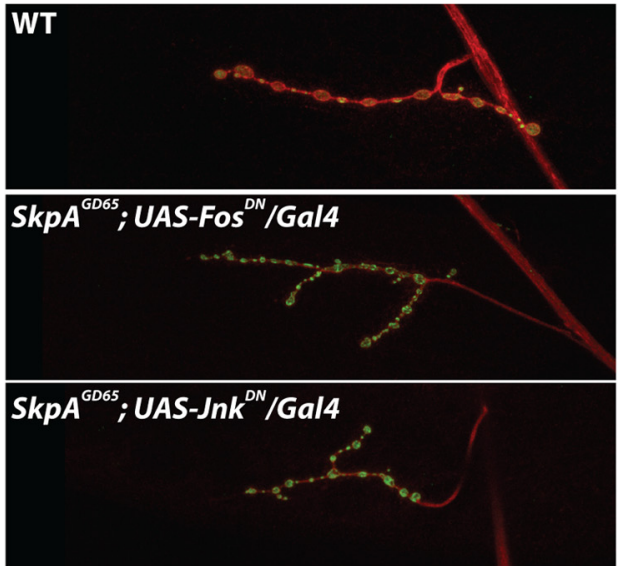
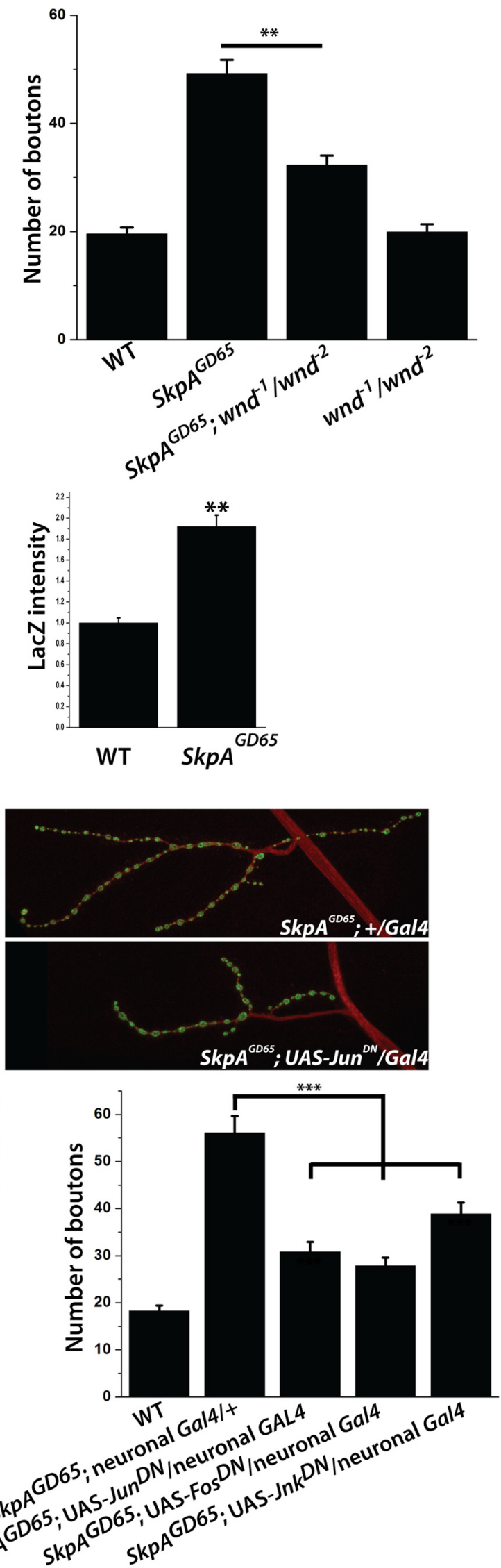

Figure 6. SkpA functions through Wnd and the Jnk MapK pathway. A, Overgrowth of the NMJ in Skp mutants requires Wnd function. Representative confocal images of muscle 4 synapses from third instar larva costained for presynaptic DVGLUT (green) and neuronal HRP (Red). Quantification of bouton number from motoneuron MN4b on musle 4 shows a significant decrease in overgrowth in the double-mutant $\left.{ }^{* * *} p<0.01\right) . N=18,18,16,17$, respectively. SkpA mutants were from homozygous mutant mothers. $\boldsymbol{B}$, puc-lacZ expression in the ventral nerve (Figure legend continues.) 
To study the effect of $S k p A$ mutants on synapse stability, we neuronally expressed an RNAi transgene targeting $\alpha$-Spectrin to induce synaptic retractions (Pielage et al., 2005). Synaptic retractions are the result of the presynaptic motoneuron terminal retracting from the postsynaptic muscle. Retractions are observed as the lack of presynaptic markers due to the loss of the presynaptic terminal. Neuronal expression of $\alpha$-spectrin RNAi led to retractions in $47 \%$ of muscle $6 / 7$ synapses (Fig. $7 A$ ). In contrast, WT animals had no synaptic retractions at muscle 6/7 synapses. When $\alpha$-spectrin RNAi was neuronally expressed in $S k p A$ mutant animals, the percentage of retractions was reduced to $5 \%$ demonstrating that SkpA is necessary for the synaptic loss. The suppression of the synaptic retraction phenotype is likely due to the upregulation of a synapse stabilization program in the absence of effective Hiw/SkpA function (Fig 7A; Massaro et al., 2009). This is consistent with a model in which the Hiw ubiquitin ligase complex selectively incorporates SkpA.

Recently, it was demonstrated that mutants in hiw and its mammalian ortholog Phrl protect synapses and axons from degeneration after axonal crush (Xiong et al., 2012; Babetto et al., 2013). Therefore, to test the role of SkpA in synaptic degeneration after injury we examined the maintenance of synapses following an axonal crush injury. To injure the axons, the segmental nerves of intact third instar larva were crushed with forceps and the larva were placed on grape plates for $20 \mathrm{~h}$ before they were dissected and stained for synaptic markers (Xiong et al., 2010). In wild-type animals, presynaptic proteins such as VGLUT disappear from the presynaptic terminal leading to innervation in only $10 \%$ of muscle 6/7 NMJs after injury (Fig. 7B). In contrast, synapses in hiw mutants are robustly maintained $20 \mathrm{~h}$ after crush as all muscle 6/7 synapses are partially or completely innervated (Fig. 7B; Xiong et al., 2012). To test whether SkpA functions in this injury response, $S k p A$ mutant larva were injured with an axonal crush and their axons were examined $20 \mathrm{~h}$ after injury. Similar to hiw mutants, $S k p A$ mutants exhibit increased synaptic maintenance with $\sim 75 \%$ of muscle $6 / 7$ synapses partially or completely innervated after injury (Fig. 7B). That mutants in hiw show stronger protection is likely due to the fact that $h i w^{\Delta N}$ is a null allele, whereas $S k p A^{G D 65}$ is a hypomorphic allele.

The above experiment focused on synaptic maintenance after injury. To visualize the role of SkpA directly in axonal degeneration we labeled single axons with GFP and compared axon fragmentation after injury. Animals expressed GFP in a small subset of motoneurons (M12-Gal4) allowing for visualization of single GFP labeled axons. Segmental nerves of third instar larva were crushed with forceps and nerves were examined $14 \mathrm{~h}$ later. In wild-type animals, the GFP labeled axons are extensively fragmented by $14 \mathrm{~h}$ after crush (Fig. 8). This is in sharp contrast to hiw mutants that display robust axon protection $14 \mathrm{~h}$ after injury (Fig. 8; Xiong et al., 2012). Similar to mutants in hiw, SkpA mutants partially inhibit axon degeneration demonstrating a role for SkpA in promoting axonal degeneration after injury (Fig. 8).

The axonal protection provided by hiw mutants is dependent on Nmnat (nicotinamide mononucleotide adenyltransferase)

\section{$\leftarrow$}

(Figure legend continued.) cord is increased almost twofold in $S k p A^{6 D 65}$ mutants compared with WT controls (WT $=1.0, n=48$ vs SkpA ${ }^{6 D 65} 1.92, n=60 ;{ }^{* *} p<0.01$ ). C, Dominant negative mutants in Fos, Jun, and Jnk suppress NMJ overgrowth phenotype. Muscle 4 synapses from third instar larva were costained for presynaptic DVGLUT (green) and neuronal HRP (red). Neuronal overexpression of each of the transgenes (UAS-Jun ${ }^{\mathrm{DN}}$, UAS-Fos ${ }^{\mathrm{DN}}$, UAS-Jnk ${ }^{\mathrm{DN}}$ ) with elav-Gal4 in combination with $S k p A^{6 D 65}$ mutant results in a significant decrease in bouton number compared with the $S k p A^{G D 65}$ mutant alone ( $\left.{ }^{* * *} p<0.001\right) . N=16,30,30,29,16$, respectively. because knockdown of Nmnat leads to increased degeneration in the hiw mutant background (Xiong et al., 2012). To determine whether this were also the case with $\operatorname{Skp} A$ mutants we expressed Nmnat RNAi in axons and tested for axonal protection. The axonal protection seen for $S k p A$ mutants is abrogated upon knockdown of Nmnat (Fig. 8). Due to the more robust protection provided by hiw mutants, we see only a modest decrease in protection from Nmnat knockdown at $14 \mathrm{~h}$ after injury, which is in agreement with the data from Xiong et al. at this time point. Hence, the axonal protection afforded by loss of highwire and $S k p A$ is likely due to the same mechanism, the maintenance of Nmnat protein and its axoprotective effect.

The delay in degeneration demonstrated above reveals a novel role for SkpA in both synaptic and axonal maintenance in addition to its role in regulating NMJ growth. This is consistent with SkpA functioning as part of an SCF-like complex with Hiw to regulate diverse neuronal functions. Hence, blocking the function of this complex is a potential therapeutic intervention for slowing axon loss resulting from injury or disease.

\section{Discussion}

The Hiw-Wnd-Jnk pathway is a central regulator of neural circuit development and maintenance. The pathway was originally studied for its role in synaptic growth and function, but also controls axon guidance, regeneration, and degeneration. Here we identify the SCF component SkpA as a component of this pathway, and demonstrate that SkpA functions to restrain synaptic terminal growth during development and promote synaptic and axonal degeneration following injury.

\section{Hiw functions with DFsn and SkpA in an atypical SCF complex}

Canonical SCF E3 ubiquitin ligases form complexes that contain Skp1, Cullin, Rbx1, and an F-Box protein that serves as the substrate adaptor. Hiw and other PHR orthologs, however, likely function as part of a novel SCF-like complex that does not include cullin and Rbx1 proteins (Saiga et al., 2009). DFsn and its orthologs, Fsn-1 in worms and Fbxo45 in mice, are the F-Box proteins that participate in the PHR complex (Liao et al., 2004; Wu et al., 2007; Saiga et al., 2009). Mutants of DFsn in Drosophila have similar phenotypes to hiw mutants, consistent with the hypothesis that the F-box protein is essential for Hiw function (Wu et al., 2007). Although Skp1 can physically interact with Rpm-1 in worms, before this study there were no functional data in any organism demonstrating a role for a Skp in PHR ligase function.

Here we present evidence that SkpA functions with Highwire to downregulate the MAP3K Wnd/DLK to restrain synaptic terminal growth. As with mutants in hiw and DFsn, mutants in $S k p A$ exhibit overgrowth of the NMJ with an increase in synaptic boutons and synaptic branch number. Consistent with SkpA and Hiw functioning in the same pathway, the NMJ overgrowth phenotype of a hiw hypomorph is dramatically enhanced in combination with a hypomorph of SkpA. As with hiw and DFsn, mutants in the ligase target Wnd/DLK as well as other components of the downstream JNK signaling pathway suppress the synaptic overgrowth phenotype of $\operatorname{skp} A$ mutants. Although the phenotypes for $S k p A$ mutants are not as strong as those seen for hiw or $D f s n$ mutants, this is likely because the $S k p A^{G D 65}$ allele analyzed here is a hypomorph. Indeed, $S k p A$ is an essential gene and homozygous null mutants are lethal (Murphy, 2003). The large size of our screen allowed us to identify a hypomorphic allele of $S k p A$ and to discover its role at the synapse. 
A
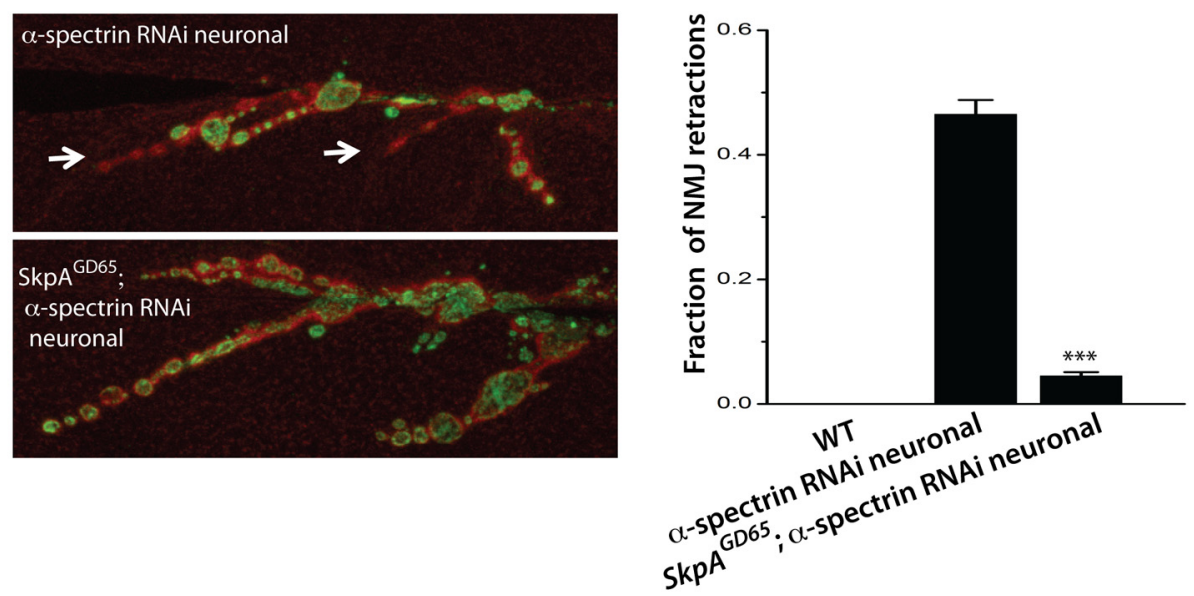

B HRP + DVGLUT HRP DVGLUT
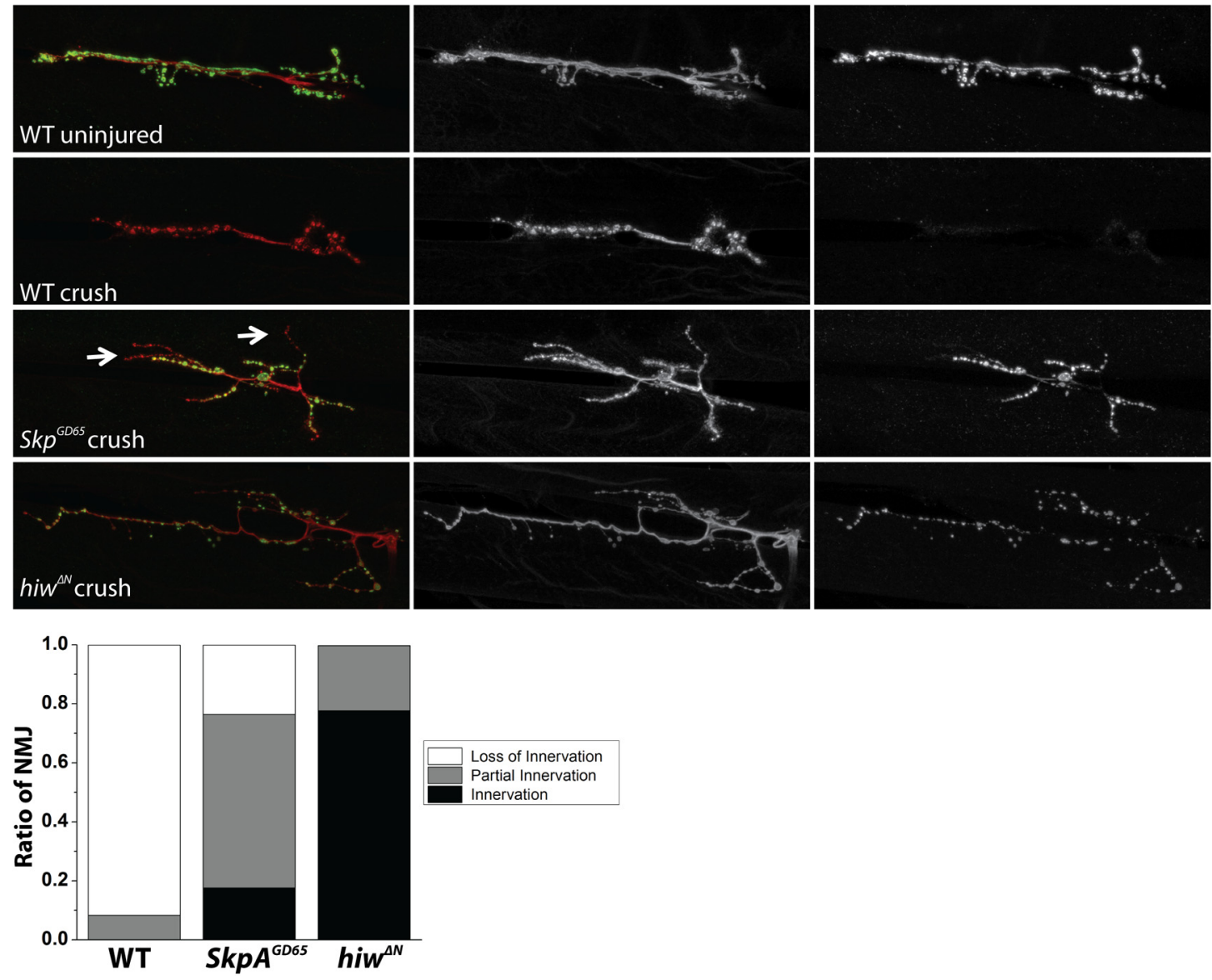

Figure 7. SkpA mutants suppress synaptic retraction. A, Representative images of muscle 6/7 NMJs stained for presynaptic DVGLUT (green) and postsynaptic DLG (red). Presynaptic knockdown of $\alpha$-spectrin results in significant synaptic retractions at NMJs. Retraction frequency is measured as the percentage of NMJs that contain DLG-positive boutons that are absent for DVGLUT staining. Retraction frequency for each genotype is shown (WT: 0 of 44; $\alpha$-spectrin RNAi: 20 of 43; SkpA ${ }^{6 D 65} ; \alpha$-spectrin RNAi: 3 of 66). $\alpha$-spectrin RNAi was expressed presynaptically by elav-Gal4. Fisher's exact test was used to determine $p$ values $\left({ }^{* *} p<0.001\right.$ ). Error bars are $95 \%$ confidence interval. $\boldsymbol{B}$, Representative images of muscle $6 / 7 \mathrm{NMJs}$ stained for presynaptic DVGLUT (green) and neuronal HRP (red). The top image is from a NMJ innervated by an uninjured axon and demonstrates DVGLUT localization throughout the boutons of WT NMJs. The bottom three images are from animals $20 \mathrm{~h}$ after axon injury by nerve crush. In wild-type animals, the presynaptic marker DVGLUT disappears from the synapse after crush. In SkpA ${ }^{6 D 65}$ mutants, the loss of DVGLUT is suppressed after crush although often with partial innervation as depicted (white arrows point out branches that are not stained for DVGLUT). hiw mutants show a more pronounced protection as $>70 \%$ of $6 / 7 \mathrm{NMJs}$ are fully innervated. Muscle $6 / 7$ NMJ's were quantified as innervated $>85 \%$, partially innervated $15-85 \%$, or loss of innervation $<15 \%$ coverage of synapse by DVGLUT; $n>17$ for each genotype.

Both hiw and $\mathrm{D} f \mathrm{~s} n$ mutants have defects in synaptic function with decreases in excitatory junction potentials (EJP) amplitude and quantal content. Although the morphological phenotype of hiw is due to activation of the Wnd pathway, the physiology defects are independent of Wnd as mutants in Wnd do not suppress the functional defect. We tested whether SkpA functions with Hiw to regulate synaptic function, or instead, whether SkpA may be selective for the targeting of Wnd and not affect the ability 

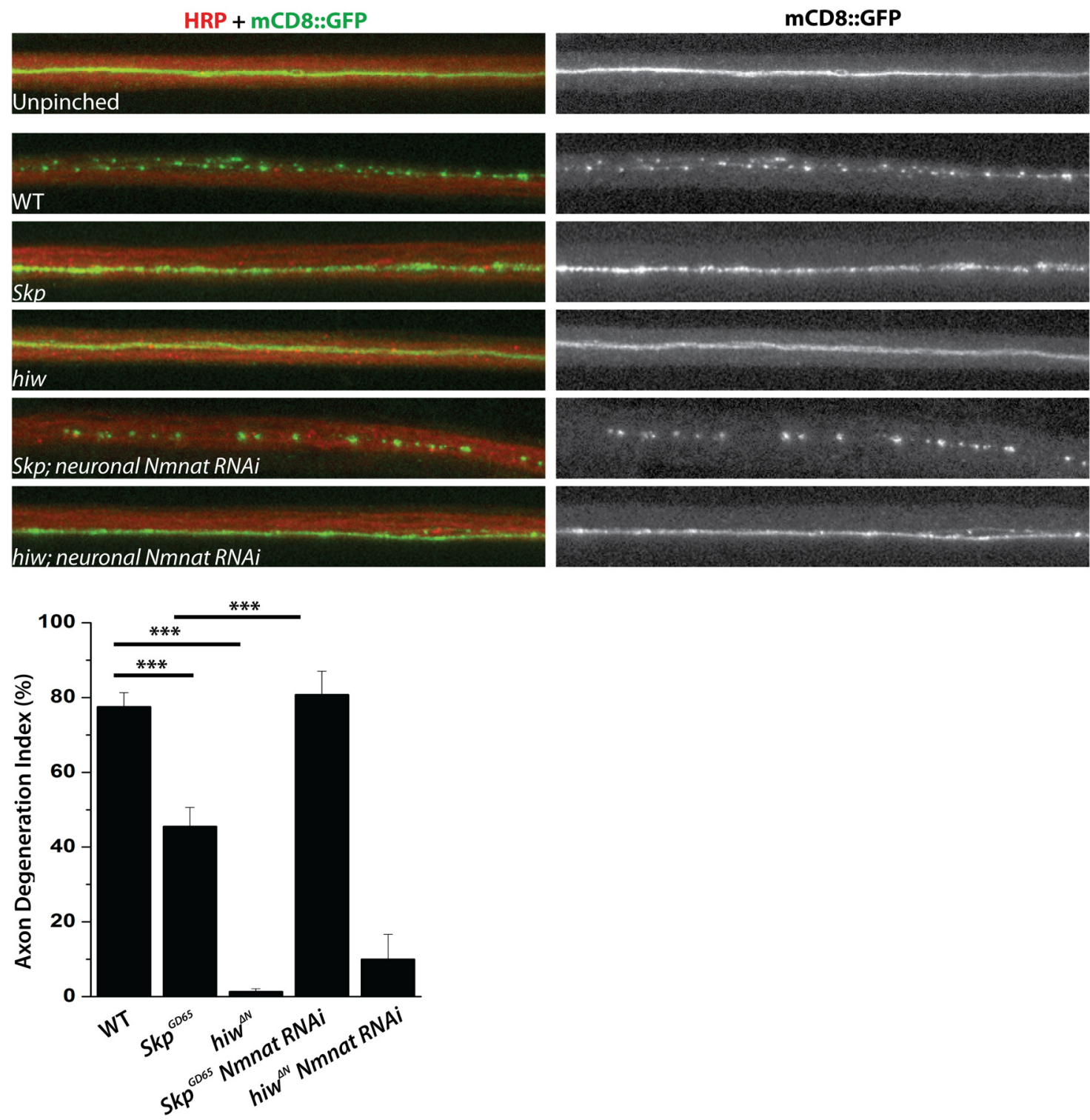

Figure 8. SkpA mutants suppress axonal degeneration after injury. The segmental nerves of third instar larva are stained by HRP (red) and single axons are labeled by the expression of UAS-mCD8:GFP by the M12-Gal4 driver. In wild-type larva, axons distal to the crush site are extensively fragmented by $14 \mathrm{~h}$ after injury as seen by fragmentation of the GFP-labeled axon. Degeneration is limited in $S k p A^{G D 65}$ and hiw ${ }^{\Delta N}$ as axons remain more intact in the mutants. Knockdown of Nmnat in the axon (M12Gal4) by RNAi blocks the ability of SkpA mutants to protect axons from degeneration. Axons were scored by researchers blind to genotype using a scale ranging from completely continuous ( $0 \%$ ), to continuous with varicosities (25\%), to partially discontinuous (50\%), to mostly fragmented with a few segments of continuity $(75 \%)$, to completely fragmented (100\%; Xiong et al., 2010). $N=69,56,55,26$, and 10 for each genotype; ${ }^{* * *} p<0.001$.

of Hiw to target the unknown substrate responsible for the physiology phenotypes. Although $S k p A^{G D 65}$ does not have a statistically significant physiology phenotype on its own, it has a pronounced decrease in EJP amplitude and quantal content when combined with a hiw hypomorph. These data demonstrate that not only does SkpA participate in the regulation of synaptic transmission, but also that SkpA functions with Hiw in this process.

SkpA promotes axonal and synaptic degeneration after injury Hiw promotes axonal and synaptic degeneration after injury (Massaro et al., 2009; Xiong et al., 2012; Babetto et al., 2013). We wondered whether SkpA selectively functions in regulating synaptic growth and function or whether it also participates in these other Hiw-dependent functions. To investigate whether SkpA promotes degeneration we tested three models of axon and synapse stability where hiw mutants provide protection. In a genetic model that results in synaptic retractions, $S k p A$ mutants provide robust protection from the loss of neuronal $\alpha$-spectrin. In addition, $S k p A$ mutants delay axonal and synaptic degeneration upon axonal injury. These findings suggest that SkpA functions with Hiw to regulate axonal stability. The ability of hiw mutants to delay axonal degeneration is likely via the increased activity of the axoprotective enzyme Nmnat, as levels of Nmnat are elevated in mutants of Drosophila hiw and mouse Phrl and knockdown of Nmnat blocks protection in both flies and mice (Xiong et al., 2012; Babetto et al., 2013). Similarly, knockdown of Nmnat abrogates axonal protection in $S k p A$ mutants, suggesting the same mechanism of action.

In vivo, mutants in Drosophila hiw or mouse Phrl provide exceptional protection from injury-induced degeneration. Mu- 
tants in hiw protect Drosophila olfactory receptor neurons for $20 \mathrm{~d}$ after axotomy (Xiong et al., 2012) and in the mouse Phr1 mutants provide robust protection for at least $10 \mathrm{~d}$ after sciatic nerve axotomy (Babetto et al., 2013). Hence, disrupting the PHR complex, including the interactions among the PHR, F-box, and Skp proteins, may be an attractive therapeutic target in neurological diseases characterized by axonal loss.

\section{References}

Aberle H, Haghighi AP, Fetter RD, McCabe BD, Magalhães TR, Goodman CS (2002) Wishful thinking encodes a BMP type II receptor that regulates synaptic growth in Drosophila. Neuron 33:545-558. CrossRef Medline

Babetto E, Beirowski B, Russler EV, Milbrandt J, DiAntonio A (2013) The Phr1 ubiquitin ligase promotes injury-induced axon self-destruction. Cell Rep 3:1422-1429. CrossRef Medline

Brace EJ, Parkinson LP, Fuller RS (2006) Skplp regulates Soi3p/Rav1p association with endosomal membranes but is not required for vacuolar ATPase assembly. Eukaryotic Cell 5:2104-2113. CrossRef Medline

Collins CA, Wairkar YP, Johnson SL, DiAntonio A (2006) Highwire restrains synaptic growth by attenuating a MAP kinase signal. Neuron 51: 57-69. CrossRef Medline

Daniels RW, Collins CA, Gelfand MV, Dant J, Brooks ES, Krantz DE, DiAntonio A (2004) Increased expression of the Drosophila vesicular glutamate transporter leads to excess glutamate release and a compensatory decrease in quantal content. J Neurosci 24:10466-10474. CrossRef Medline

DiAntonio A, Haghighi AP, Portman SL, Lee JD, Amaranto AM, Goodman CS (2001) Ubiquitination-dependent mechanisms regulate synaptic growth and function. Nature 412:449-452. CrossRef Medline

Eresh S, Riese J, Jackson DB, Bohmann D, Bienz M (1997) A CREB-binding site as a target for decapentaplegic signalling during Drosophila endoderm induction. EMBO J 16:2014-2022. CrossRef Medline

Hammarlund M, Nix P, Hauth L, Jorgensen EM, Bastiani M (2009) Axon regeneration requires a conserved MAP kinase pathway. Science 323:802806. CrossRef Medline

Huntwork-Rodriguez S, Wang B, Watkins T, Ghosh AS, Pozniak CD, Bustos D, Newton K, Kirkpatrick DS, Lewcock JW (2013) JNK-mediated phosphorylation of DLK suppresses its ubiquitination to promote neuronal apoptosis. J Cell Biol 202:747-763. CrossRef Medline

Juo P, Kaplan JM (2004) The anaphase-promoting complex regulates the abundance of GLR-1 glutamate receptors in the ventral nerve cord of $C$. elegans. Curr Biol 14:2057-2062. CrossRef Medline

Liao EH, Hung W, Abrams B, Zhen M (2004) An SCF-like ubiquitin ligase complex that controls presynaptic differentiation. Nature 430:345-350. CrossRef Medline

Martín-Blanco E, Gampel A, Ring J, Virdee K, Kirov N, Tolkovsky AM, Martinez-Arias A (1998) Puckered encodes a phosphatase that mediates a feedback loop regulating JNK activity during dorsal closure in Drosophila. Genes Dev 12:557-570. CrossRef Medline

Massaro CM, Pielage J, Davis GW (2009) Molecular mechanisms that enhance synapse stability despite persistent disruption of the spectrin/ ankyrin/microtubule cytoskeleton. J Cell Biol 187:101-117. CrossRef Medline

Marrus SB, DiAntonio A (2004) Preferential localization of glutamate receptors opposite sites of high presynaptic release. Curr Biol 14:924-931. CrossRef Medline

Miller BR, Press C, Daniels RW, Sasaki Y, Milbrandt J, DiAntonio A (2009) A dual leucine kinase-dependent axon self-destruction program promotes Wallerian degeneration. Nat Neurosci 12:387-389. CrossRef Medline

Murphy TD (2003) Drosophila skpA, a component of SCF ubiquitin ligases, regulates centrosome duplication independently of cyclin E accumulation. J Cell Sci 116:2321-2332. CrossRef Medline

Nakata K, Abrams B, Grill B, Goncharov A, Huang X, Chisholm AD, Jin Y (2005) Regulation of a DLK-1 and p38 MAP kinase pathway by the ubiquitin ligase RPM-1 is required for presynaptic development. Cell 120: 407-420. CrossRef Medline

Pielage J, Fetter RD, Davis GW (2005) Presynaptic spectrin is essential for synapse stabilization. Curr Biol 15:918-928. CrossRef Medline
Po MD, Hwang C, Zhen M (2010) PHRs: bridging axon guidance, outgrowth and synapse development. Curr Opin Neurobiol 20:100-107. CrossRef Medline

Rigaut G, Shevchenko A, Rutz B, Wilm M, Mann M, Séraphin B (1999) A generic protein purification method for protein complex characterization and proteome exploration. Nat Biotechnol 17:1030-1032. CrossRef Medline

Ritzenthaler S, Suzuki E, Chiba A (2000) Postsynaptic filopodia in muscle cells interact with innervating motoneuron axons. Nat Neurosci 3:10121017. CrossRef Medline

Saiga T, Fukuda T, Matsumoto M, Tada H, Okano HJ, Okano H, Nakayama KI (2009) Fbxo45 forms a novel ubiquitin ligase complex and is required for neuronal development. Mol Cell Biol 29:3529-3543. CrossRef Medline

Sanyal S, Narayanan R, Consoulas C, Ramaswami M (2003) Evidence for cell autonomous AP1 function in regulation of Drosophila motor-neuron plasticity. BMC Neurosci 4:20. CrossRef Medline

Schaefer AM, Hadwiger GD, Nonet ML (2000) Rpm-1, a conserved neuronal gene that regulates targeting and synaptogenesis in C. elegans. Neuron 26:345-356. CrossRef Medline

Schulman BA, Carrano AC, Jeffrey PD, Bowen Z, Kinnucan ER, Finnin MS, Elledge SJ, Harper JW, Pagano M, Pavletich NP (2000) Insights into SCF ubiquitin ligases from the structure of the Skp1-Skp2 complex. Nature 408:381-386. CrossRef Medline

Shin JE, Cho Y, Beirowski B, Milbrandt J, Cavalli V, DiAntonio A (2012) Dual leucine zipper kinase is required for retrograde injury signaling and axonal regeneration. Neuron 74:1015-1022. CrossRef Medline

Stewart BA, Atwood HL, Renger JJ, Wang J, Wu CF (1994) Improved stability of Drosophila larval neuromuscular preparations in haemolymph-like physiological solutions. J Comp Physiol A 175:179_ 191. Medline

Tian X, Li J, Valakh V, DiAntonio A, Wu C (2011) Drosophila Rael controls the abundance of the ubiquitin ligase Highwire in post-mitotic neurons. Nat Neurosci 14:1267-1275. CrossRef Medline

Tian X, Zhu M, Li L, Wu C (2013) Identifying protein-protein interaction in Drosophila adult heads by tandem affinity purification (TAP). J Vis Exp 82:50968. CrossRef Medline

Valakh V, Walker LJ, Skeath JB, DiAntonio A (2013) Loss of the spectraplakin short stop activates the DLK injury response pathway in Drosophila. J Neurosci 33:17863-17873. CrossRef Medline

Wan HI, DiAntonio A, Fetter RD, Bergstrom K, Strauss R, Goodman CS (2000) Highwire regulates synaptic growth in Drosophila. Neuron 26: 313-329. CrossRef Medline

Watkins TA, Wang B, Huntwork-Rodriguez S, Yang J, Jiang Z, EasthamAnderson J, Modrusan Z, Kaminker JS, Tessier-Lavigne M, Lewcock JW (2013) DLK initiates a transcriptional program that couples apoptotic and regenerative responses to axonal injury. Proc Natl Acad Sci U S A 110:4039-4044. CrossRef Medline

Weber U, Paricio N, Mlodzik M (2000) Jun mediates frizzled-induced $\mathrm{R} 3 / \mathrm{R} 4$ cell fate distinction and planar polarity determination in the Drosophila eye. Development 127:3619-3629. Medline

Welsbie DS, Yang Z, Ge Y, Mitchell KL, Zhou X, Martin SE, Berlinicke CA, Hackler L Jr, Fuller J, Fu J, Cao LH, Han B, Auld D, Xue T, Hirai S, Germain L, Simard-Bisson C, Blouin R, Nguyen JV, Davis CH, et al. (2013) Functional genomic screening identifies dual leucine zipper kinase as a key mediator of retinal ganglion cell death. Proc Natl Acad Sci U S A 110:4045-4050. CrossRef Medline

Wu C, Wairkar YP, Collins CA, DiAntonio A (2005) Highwire function at the Drosophila neuromuscular junction: spatial, structural, and temporal requirements. J Neurosci 25:9557-9566. CrossRef Medline

Wu C, Daniels RW, DiAntonio A (2007) DFsn collaborates with Highwire to down-regulate the Wallenda/DLK kinase and restrain synaptic terminal growth. Neural Dev 2:16. CrossRef Medline

Xiong X, Wang X, Ewanek R, Bhat P, Diantonio A, Collins CA (2010) Protein turnover of the Wallenda/DLK kinase regulates a retrograde response to axonal injury. J Cell Biol 191:211-223. CrossRef Medline

Xiong X, Hao Y, Sun K, Li J, Li X, Mishra B, Soppina P, Wu C, Hume RI, Collins CA (2012) The Highwire ubiquitin ligase promotes axonal degeneration by tuning levels of Nmnat protein. PLoS Biol 10:e1001440. CrossRef Medline

Yan D, Jin Y (2012) Regulation of DLK-1 kinase activity by calcium- 
mediated dissociation from an inhibitory isoform. Neuron 76:534-548. CrossRef Medline

Yan D, Wu Z, Chisholm AD, Jin Y (2009) The DLK-1 kinase promotes mRNA stability and local translation in C. elegans synapses and axon regeneration. Cell 138:1005-1018. CrossRef Medline

Yao KM, White K (1994) Neural specificity of elav expression: defining a Drosophila promoter for directing expression to the nervous system. J Neurochem 63:41-51. CrossRef Medline

Yoshimura K, Ueno M, Lee S, Nakamura Y, Sato A, Yoshimura K, Kishima H, Yoshimine T, Yamashita T (2011) c-Jun N-terminal kinase induces axonal degeneration and limits motor recovery after spinal cord injury in mice. Neurosci Res 71:266-277. CrossRef Medline
Zhang YQ, Bailey AM, Matthies HJ, Renden RB, Smith MA, Speese SD, Rubin GM, Broadie K (2001) Drosophila fragile X-related gene regulates the MAP1B homolog Futsch to control synaptic structure and function. Cell 107:591-603. CrossRef Medline

Zhen M, Huang X, Bamber B, Jin Y (2000) Regulation of presynaptic terminal organization by C. elegans RPM-1, a putative guanine nucleotide exchanger with a RING-H2 finger domain. Neuron 26:331-343. CrossRef Medline

Zheng N, Schulman BA, Song L, Miller JJ, Jeffrey PD, Wang P, Chu C, Koepp DM, Elledge SJ, Pagano M, Conaway RC, Conaway JW, Harper JW, Pavletich NP (2002) Structure of the Cul1-Rbx1-Skp1-F boxSkp2 SCF ubiquitin ligase complex. Nature 416:703-709. CrossRef Medline 